\title{
Design Guidelines and Manufacturing Methods for Microreaction Devices
}

\author{
Wolfgang Ehrfeld*
}

\begin{abstract}
Microreaction technology is a powerful tool for the evaluation of new reaction pathways, process intensification in chemical engineering and combinatorial material development. Because of the small characteristic dimensions of microreaction devices, mass and heat transfer processes are enhanced and, in addition, initial and boundary conditions can be precisely adjusted for optimizing yield and selectivity. A number of microfabrication methods exist to cover prototyping and manufacturing of a few items as well as costeffective mass production of modular and integrated microdevices for unit operations, reactions and control. Detailed analyses of methods like LIGA technology, wet and dry micro-etching, mechanical micromachining and laser processing show that a wide variety of materials is applicable to meet most requirements of chemical processes.
\end{abstract}

Keywords: Microfabrication · Microreactors · Process intensification · Unit operation modules

\section{Miniaturization of Chemical Pro- cessing Units}

During the past fifty years, general technological progress has been dominated essentially by a unique strategy of success which constantly aims at comprehensive miniaturization and integration of functional elements in technical systems. The most outstanding development took place in microelectronics where, in the meantime, integrated circuits with hundreds of millions or even billions of transistors have become products of our daily lives. More recently, micromechanical, microoptical, microfluidic, and many other microdevices have become the basis for a further multibillion dollar business, the market of microtechnology [1-3].

Researchers in chemical engineering and the life sciences are also intensively analyzing the possibilities which are potentially offered by modern technologies for miniaturization and integration [4-7].

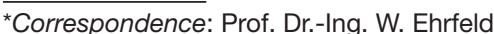
Ehrfeld Mikrotechnik AG

Mikroforum Ring 1

D-55234 Wendelsheim, Germany

Tel.: + 496734919300

Fax: + 496734919305

E-Mail: wolfgang.ehrfeld@ehrfeld.com

www.ehrfeld.com
These technologies have created a novel basis

- to realize extremely powerful tools for evaluation of new reaction pathways,

- to implement comprehensively the concept of process intensification, and

- to accelerate screening in combinatorial material development.

As a matter of fact, the development status of microreaction technology is still very much in its infancy. There are many reservations about technical feasibility, life time of process units, fouling problems and, in particular, productivity and investment costs. The acceptance problems of microreaction technology correspond essentially to those of nearly all novel developments in modern technologies [8].

Nevertheless, there are also convincing arguments for utilizing miniaturization and integration in modern chemistry and life sciences. Microdevices for chemical processing are evidently characterized by a small holdup. When using proper materials, they withstand high pressures and temperatures and, consequently, they can be safely operated within a wide range of operating conditions and a nearly inherent safety for chemical plants seems to be achievable. Due to the small dimensions extremely high heat and mass transfer rates can be utilized so that defined reaction conditions can be precisely adjusted. In addition, the response time of a miniaturized system is usually much smaller than that of a macrosystem so that chemical processes become easily controllable. Evidently, microreaction devices are powerful tools for process development and in particular, for the evaluation of new reaction pathways usually not accessible by standard laboratory equipment.

Process intensification aims at a dramatic reduction in plant size without decreasing the production capacity [9-11]. By this means much longer investment, operating and maintenance costs should be achievable. A straightforward approach to meet these requirements is to replace batch processing by continuous operation. Problems in terms of safety and process control often connected with continuous processes can be solved by microreaction devices and also dangerous run-away effects play no essential role in miniaturized systems. The effects of more intensive heat and mass transfer, more precise process control or access to novel process routes contribute additionally to higher yield and selectivity of chemical reactions and, correspondingly, to the cost reduction target of the process intensification strategy. Furthermore microreaction devices allow the concept of numbering-up to be applied instead of scale-up and hence improve the flexibility of a plant. Obviously, the ultimate development of 
process intensification leads to microreaction technology [12].

Combinatorial material development is based on highly effective synthesizing and screening of a huge number of chemical compounds. By means of microreaction devices not only the amount of reactants, auxiliary substances, waste, energy and space can be minimized but also all the other advantages of microreaction devices mentioned above can be favorably utilized [13][14]. Consequently, the concept of microreaction technology has been implemented extremely successfully since a fairly long time in combinatorial material development comprising e.g. research on new drugs or more efficient catalysts.

However, microfabrication methods, which are usually not familiar to chemical engineers, have to be introduced to enable them to profit comprehensively from microreaction technology. This transition from standard manufacturing methods of plant components to development and production of microdevices is also inevitably connected with the application of special materials which are not yet proven in chemical engineering. In addition, novel design rules which do not exist up to now should be implemented for the long term to speed up the development of novel devices. In the following, these aspects will be discussed in more detail.

\section{From Basic Properties to Technical Design Rules}

\subsection{Status of Development}

In contrast to microelectronics where extremely powerful software and detailed design rules exist for the development of ultra-large-scale integrated circuits, there are no corresponding comprehensive tools in microreaction technology available to date. Such design tools should comprise mathematical modeling of flow and chemical reactions in miniaturized systems as well as specifications for suitable materials and simulation of manufacturing processes applicable for the respective microreaction devices.

Since it will take several years to realize such an integral software tool box, individual approaches with separate steps have to be applied to meet gradually the requirements of microreactor design. Standard software for computational fluid dynamics is directly applicable in this context and there are also powerful software tools for the simulation of special steps of microfabrication processes while rather poor experience exists in view of materials for microreactors, optimization of microreactor design or treatment of interdependent effects. Consequently, a profound knowledge of the basic properties and phenomena of microreaction technology is absolutely essential for a successful design of microreaction devices. Some of the major effects are summarized in the following.

\subsection{Molecular Transport Phenomena}

Diffusion, thermal conductivity, and viscosity are physically similar phenomena which involve the transport of a physical quantity through a gas or liquid. The driving forces for the corresponding transport fluxes of mass, energy and momentum are the gradients in concentration, temperature and velocity, respectively, where in all three cases the fluxes are in the same direction as the gradients. For given differences in these properties, a decrease in the characteristic dimensions results in an increase of these gradients and, correspondingly, in mass and heat transfer rates as well as in viscous losses.

Proper design rules must take into account that mixing and heat exchange systems with extremely high transfer rates per unit volume can be realized by miniaturization but an increase in losses due to viscosity may counterbalance the positive effects. Accordingly, suitable figures of merit must be defined for micromixers and micro heat exchangers which consider, for instance, the ratio of mass or heat fluxes to pressure losses. However, the value of such a figure of merit should be always considered in context with further boundary conditions of the process. Decreasing the characteristic dimensions of a system results e.g. in a reduction of the material hold-up and a simultaneous enlargement of the surface area to volume ratio of the system. These aspects determine additionally the speed of mixing and heat transfer and, consequently, the degree of miniaturization required in a specific case which has to be considered in view of a favorable design for a microreaction system.

It was meanwhile demonstrated in many cases that highly exothermal reactions can be performed under isothermal conditions using the channels of micro heat exchangers as reaction volumes [15]. Micromixers allow mixing times in the submillisecond range to be achieved. In addition, gas-liquid suspensions and liquidliquid emulsions with extremely small bubble and droplet sizes, respectively, and high uniformity can be generated by means of such devices [16]. Accordingly, micromixers are promising tools to improve the performance of phase transfer and other exchange processes.

\subsection{Transport of Bulk Material}

As a matter of fact, problems also arise regarding the design of microreaction systems. For instance, gravitational forces cannot be efficiently utilized to transport fluids at small characteristic dimensions since the effects of surface forces exceed by far those of mass or bulk forces. This is immediately evident when regarding the reflux in a distillation column or the settler in a mixer settler system. If characteristic dimensions in the millimeter range would be applied the operating conditions and performance of such systems should be changed considerably and alternative design concepts would be required.

Consequently, the design of miniaturized systems must aim at alternative methods for phase separation like microfiltration to break emulsions, utilization of hydrophobic and hydrophilic surfaces, application of high centrifugal forces, etc. If large amounts of materials have to be transported, a favorable design should consider large pumps rather than arrangements with many micropumps which, in most cases, are commercially not attractive for cost reasons and technically less suitable because of their comparatively low efficiency.

\subsection{Adjustment of Reaction \\ Conditions}

The extreme enhancement in mass and heat transfer rates and the reduction of hold-up results in fundamentally novel design possibilities in view of selecting alternative reaction routes. In contrast to macro devices like large stirring tanks, the starting conditions for a chemical reaction can be set precisely in respect of time and concentration because of much faster mixing of educts in a micromixer. The reaction starts at a precisely defined position with a spatially uniform composition. Consequently, unfavorable reaction conditions due to incomplete mixing, which eventually result in undesired side and secondary reactions and, consequently, losses in yield and selectivity, are minimized.

The high heat transfer rates achievable in micro heat exchangers and reactors allow unfavorable reaction conditions resulting from hot spots or thermal runaway effects to be avoided. An optimum temperature or temperature profile for the reaction can be chosen in respect of spatial distribution and time. Thus, a fast flowing fluid element can be cooled down or heated up very rapidly in fractions of a second and, because of the small thermal mass of microdevices, a periodic change of temperature of the reactor can be realized with a typical time constant of some seconds. All these examples offer possibilities to design microreaction sys- 
tems with high yield and selectivity.

An inherent problem, however, is the adjustment of residence time when narrow channels or capillaries are used as delay loops. Because of the low Reynolds number a viscous laminar flow exists which, due to its velocity profile, results in a correspondingly broad distribution of residence time.

\subsection{Process Control and Safety}

The inherent advantage of precise adjustment of starting and boundary conditions for chemical reactions and unit operations in microdevices provides a novel basis for process control. Taking into account in addition the small hold-up, it is evident that an extremely short response time is a further inherent advantage of microreaction devices in view of process control. There is a unique chance to design microreaction systems for novel reaction routes which were not applied so far commercially for reasons of safety, difficulties in process control or because it is fundamentally impossible to apply such routes in macroscopic devices.

This is the case in particular for controlled reactions in the explosive regime [17]. This becomes accessible by means of microreaction devices since they can be designed to act like flame retention baffles. Moreover, these prerequisites allow reactions to be performed at an extremely high pressure level which is of importance for chemical processes using supercritical solvents. As a result, a nearly inherent safety is achievable by means of microreaction technology if proper design and operation concepts are applied.

\subsection{The Numbering-up Concept}

The safety problems associated with the material hold-up within the operating units of a chemical plant may be reduced essentially by applying microreaction devices. The problems resulting from storage of large quantities of educts and products remain, of course, unchanged when a conventional plant is replaced by a microreaction plant with the same production capacity. Nevertheless, this problem may be reduced by replacing a large plant by several small plants for distributed production on site and on demand. In contrast to conventional plants with macroscopic process devices where scale-up usually results in a considerable reduction of specific investment costs, microreaction plants may rather profit from mass production of microdevices in order to reduce specific investment costs. Scale-up to achieve the desired production capacity can be done only at one site while, according to the numbering-up concept, a plant comprising a large number of chemical microdevices can be split for production at several sites. Evidently, aspects of cost effective series production have to be strictly regarded in the framework of microreactor design.

There are a number of further advantages of the numbering-up concept. Research results can be more quickly transferred into production, plants can be constructed in a shorter time and the production capacity can be adjusted more flexibly to variations in demand. Since mass production of microdevices may result in relatively low costs per piece, novel design concepts for cost saving maintenance and repair based on disposable elements might be introduced.

\section{Microfabrication of Reaction and Unit Operation Devices}

\subsection{General Requirements}

Since the production of chemicals in a continuous process is inevitably connected with a transport of material, three-dimensional microfabrication processes are required in order to realize sufficiently large cross-sections for channels and ducts as well as reaction volumes. Meanwhile, a wide variety of such processes as well as design and test methods exist which all essentially originated either from semiconductor technology or precision engineering. Thin film methods applied to a large extent in semiconductor technology are less suitable for the generation of three-dimensional microreaction devices but are widely used for surface processing and protection as well as for manufacturing of sensor elements.

According to the extremely wide variety of reactions, educts, products, and process conditions, a sufficiently broad spectrum of materials is required to realize suitable microdevices for chemical processes. Metals and metal alloys, plastics, glass, ceramic materials, semiconductor materials like silicon and various auxiliary materials for sealing, surface treatment, etc. have been in the meantime successfully applied for the realization of microreaction devices.

Besides such basic aspects concerning shape and materials of microreaction devices, costs play a major role in the selection of a microfabrication process. In this respect, the number of pieces and the precision which is really required as well as aspects like availability, manufacturing experience etc. must be taken into account. In contrast to the situation some years ago the prerequisites for cost-effective mass fabrication as well as small-scale production or rapid prototyping have changed essentially. In the meantime modern commercial equipment for production of microdevices has become available which allows the replacement of unreliable and uneconomic laboratory-scale manufacturing devices.

Mathematical modeling of the device function may also help essentially to save costs since it allows more realistic specifications to be established with regard to functional requirements. In addition, mathematical modeling of the process sequence for microfabrication and assembly will be useful for cost saving. Such hard and soft aspects will be considered in some more detail in the following analysis of microfabrication methods for reaction devices.

\subsection{LIGA Technology}

The LIGA technology allows the production of ultra precise microstructures with extreme aspect ratio from a wide variety of materials [12][18]. It is based on a combination of deep lithography, electroforming and molding processes. In the first step of the manufacturing sequence, a pattern from a mask or by means of a serial beam writing process is transferred into a thick resist layer on an electrically conductive substrate. Ultra precise microstructures with extreme aspect ratio can be generated by deep X-ray lithography. Using special epoxy resists like SU 8, which utilize intrinsic optical wave guide properties of irradiated cross-linked regions, favorable results are also achievable by means of UV lithography. In the second step, the threedimensional relief-like structure of the resist polymer generated by deep lithography is transferred into a complementary metallic structure by means of electroforming starting from the electrically conductive substrate. This metal structure may be the final product in some special cases.

In general, however, the metal structure is used in a third step as a master tool for a replication process like injection molding, casting or embossing for mass fabrication of microstructures. A wide variety of mold materials can be applied for micromolding, e.g. organic polymers, pre-ceramic polymers, ceramic and metallic powders with organic binders for subsequent sintering etc. so that most material requirements for chemical microdevices can be favorably met.

Today, there are a number of LIGA products which evidently have promising markets in the fields of microoptics and integrated optics, molecular biotechnology, and microactuators. More recently, LIGA components and systems have been successfully applied to chemical engineering and microreaction technology, respectively. 
A number of chemical companies and, of course, research institutes now utilize such devices like micromixers, micro heat exchangers, and micro bubble columns as well as modular systems with integrated functional elements for reaction, heat transfer, mixing, separation and fluid distribution for process development (Fig. 1). LIGA devices are also seriously considered for the production of fine chemicals by chemical industry.

\subsection{Wet and Dry Etching Processes}

Wet etching processes are widely used to produce microstructures by means of transferring resist patterns into various materials. However, for most materials only isotropic etching processes exist so that, because of lateral under-etching of the resist pattern, only shallow micro channels or other shallow structures can be generated at the surface of a bulk material. Threedimensional structures can be manufactured when the pattern is etched completely through thin foils which have then to be stacked in order to realize deep micro channels with high aspect ratio.

Isotropic etching has been applied several times for manufacturing microreaction devices. The technological expenditure is relatively low but there are some restrictions concerning accuracy, surface roughness and geometrical design. The product spectrum comprises various types of heat exchangers, micromixers, separators, reaction units and even integrated devices with several functional elements (Fig. 2).

Wet chemical anisotropic etching of monocrystalline silicon has been widely applied in microtechnology [19]. This method is based on the dependence of etching velocity on crystal orientation so that only a few basic geometries can be realized. Besides silicon, only very limited manufacturing experience exists with other monocrystalline, inevitably very expensive, materials. Consequently, wet chemical anisotropic etching is in general not very attractive for manufacturing chemical microdevices because of strong restrictions in respect of shape and material. Nevertheless, the technological expenditure is low and material problems can also be solved by deposition of protection layers. A number of microfluidic devices have been manufactured by means of this method such as micropumps, microvalves and flow distribution systems.

Besides anisotropic etching of monocrystalline materials a further wet chemical etching process exists which uses a special type of photo-sensitive glass [20]. A wafer consisting of such glass is irradiated through a mask with UV light and subse-

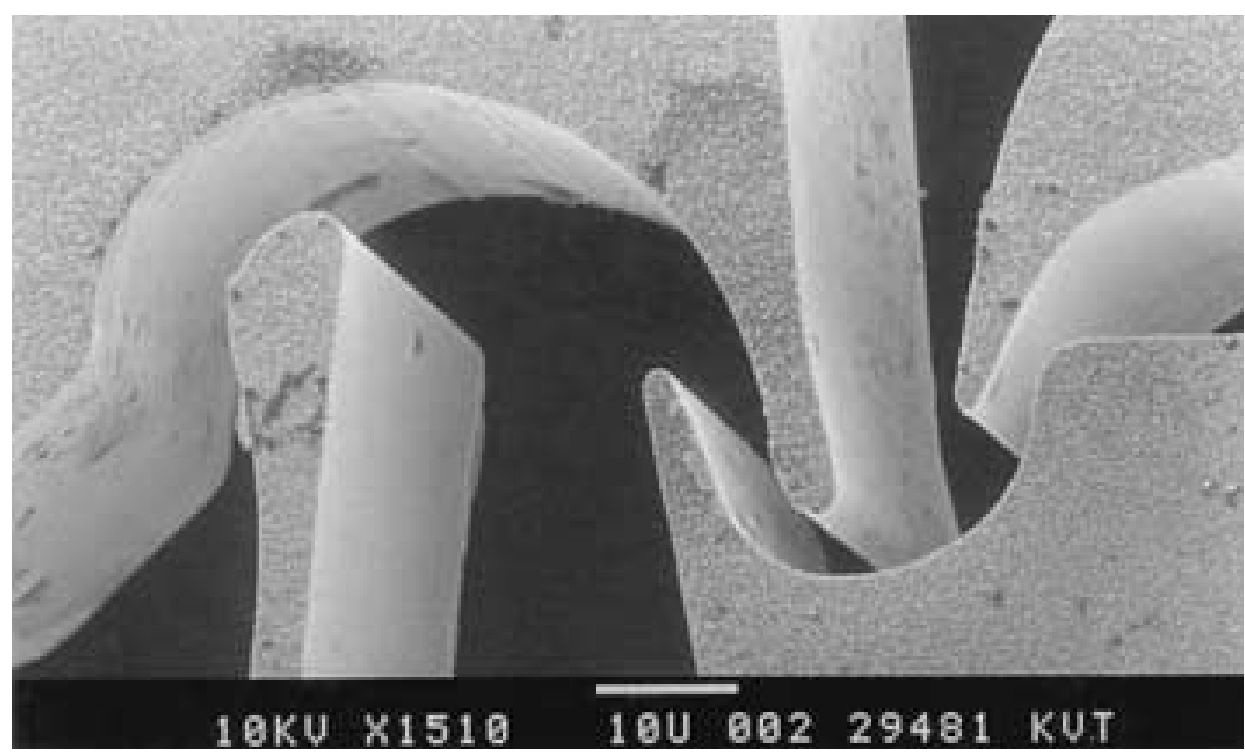

Fig. 1. Double deflecting micro nozzle for aerodynamic separation of uranium isotopes manufactured by LIGA technology from nickel. The smallest characteristic dimensions achieved in such devices is below $10 \mu \mathrm{m}$. (Source: Institute of Nuclear Process Engineering at the former Karlsruhe Nuclear Research Center - now Forschungszentrum Karlsruhe, Siemens)

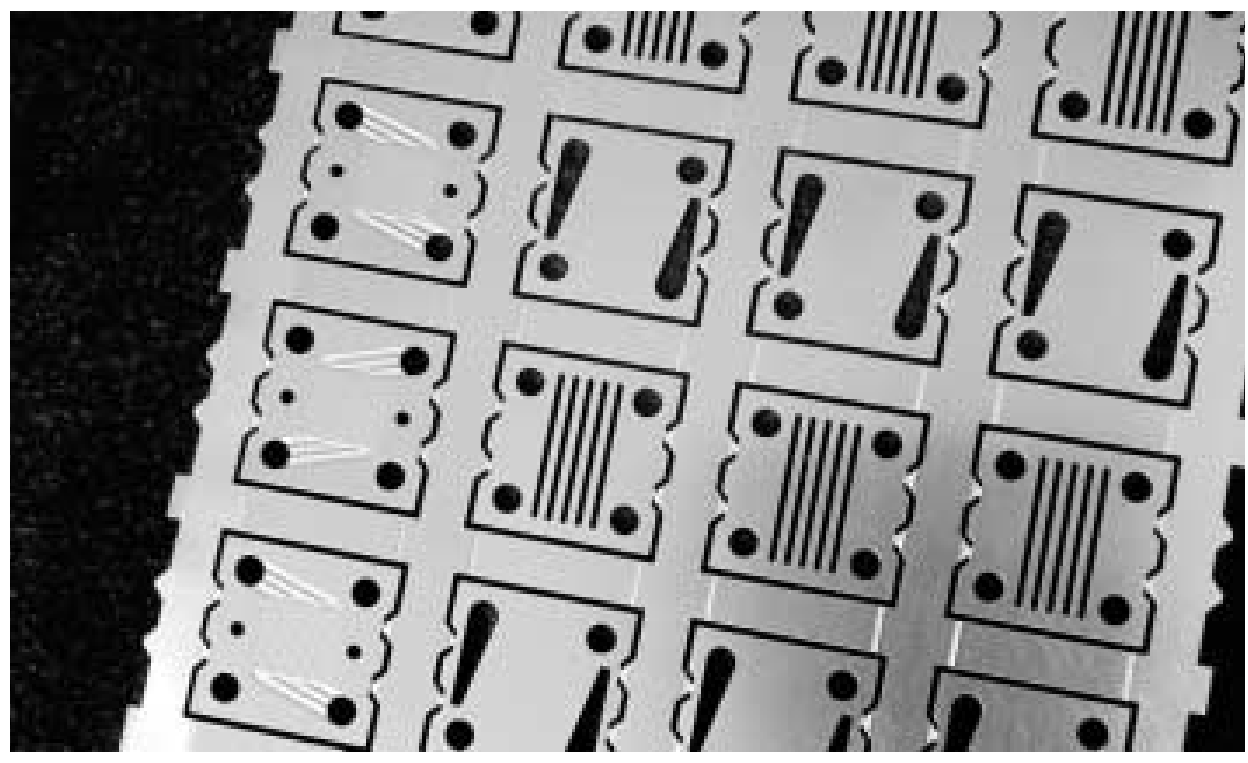

Fig. 2. Microetched foil of stainless steel for manufacturing micro heat exchangers by stacking and diffusion welding (Source: Ehrfeld Mikrotechnik, Ätztechnik Herz)

quently heated to a temperature between 800 and $900 \mathrm{~K}$. This results in crystallization of the irradiated regions which can then be dissolved much faster in hydrofluoric acid than the non-irradiated parts. Meanwhile, this method has been successfully applied to produce microreaction devices like mixers, heat exchangers and micro titer plates from glass.

Precise microstructures with nearly any cross-sectional shape can be generated by means of anisotropic plasma etching methods where again silicon is the most important and proven material [19][21]. Usually, a mask pattern is transferred into a thin layer consisting of a material resistant against plasma etching on a silicon wafer. Subsequently silicon is etched by means of a fluorine-containing low pressure plasma which generates gaseous silicon compounds.

In order to generate microstructures with extremely high aspect ratio the directed etching process is connected with a subsequent deposition process from the plasma where the walls oriented in parallel to the etching direction are covered with plasma polymer resistant against the reactive plasma [22]. By means of multiple repetition of 


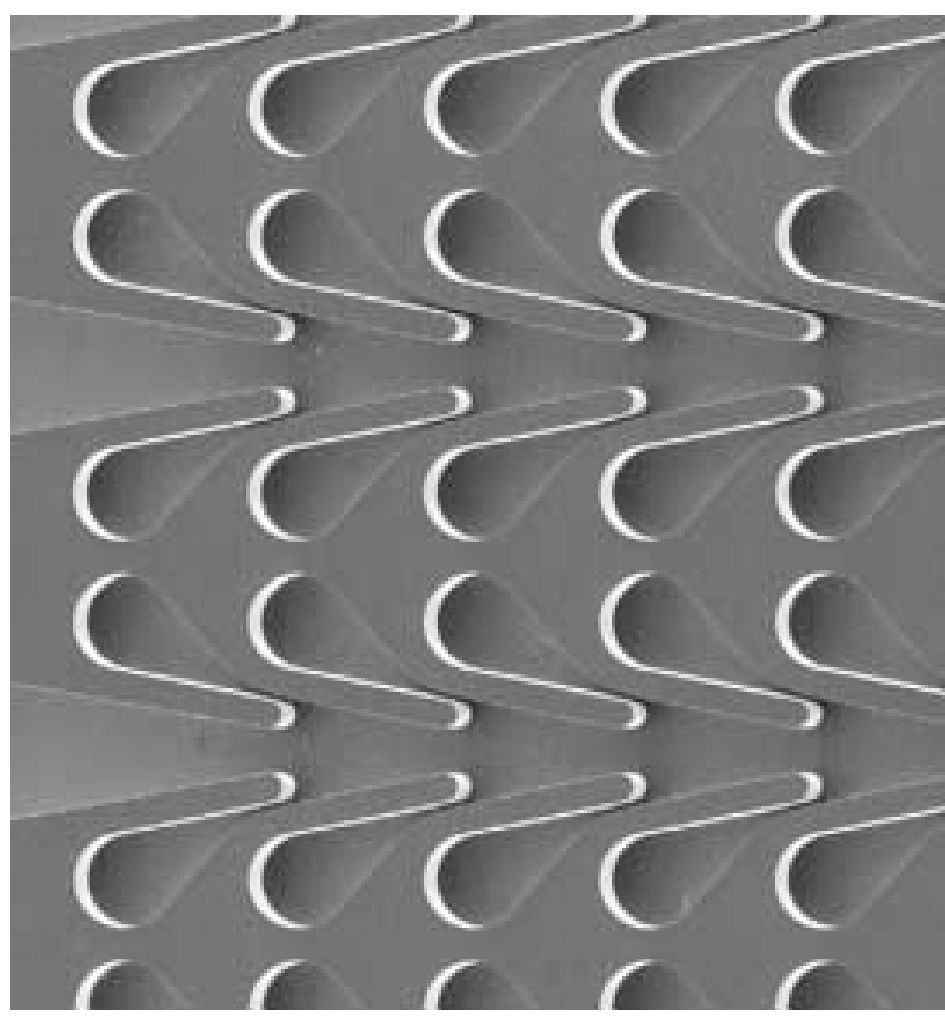

Fig. 3. View on the channel structure of a phase separator generated by ASE deep etching of silicon (Source: IMM)

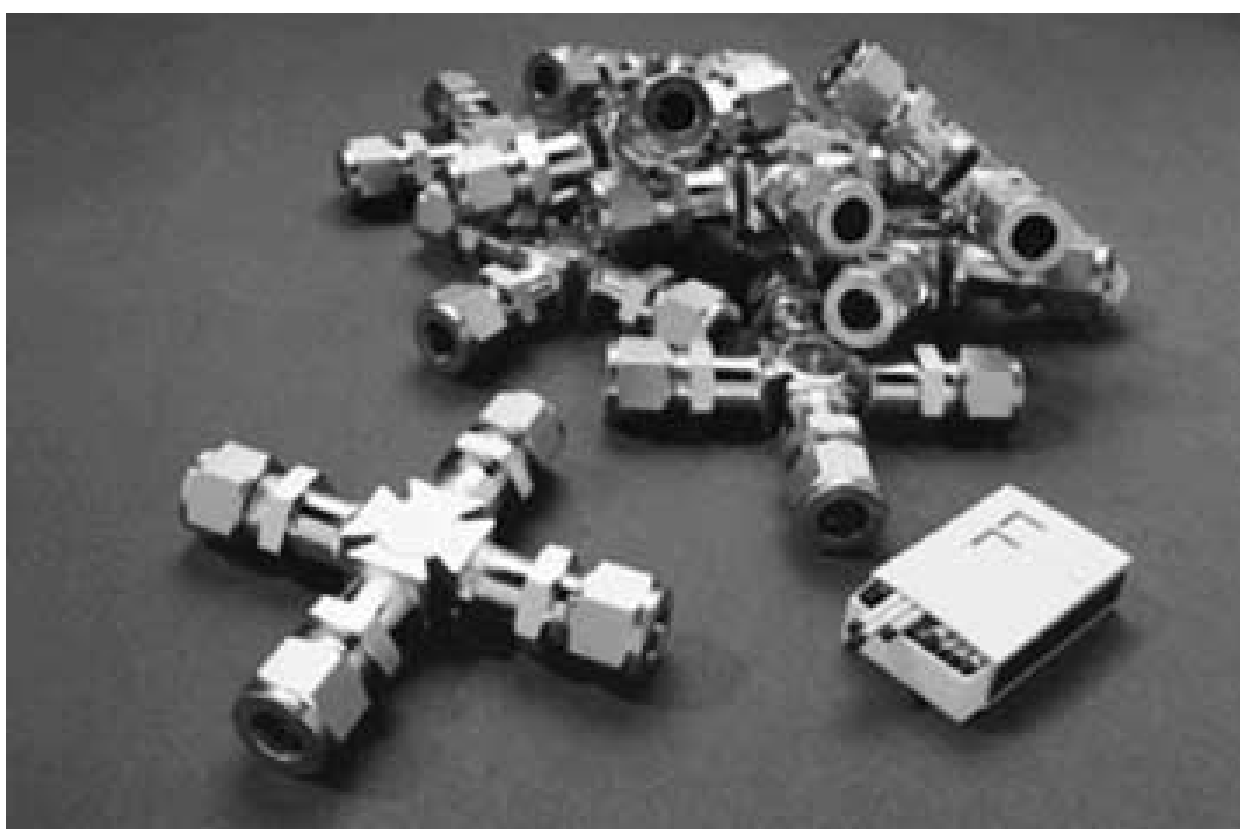

Fig. 4. Micro heat exchangers manufactured by mechanical micromachining (Source: Forschungszentrum Karlsruhe)

directed etching and side wall passivation channels and other structures with nearly vertical walls can be realized and, accordingly, extremely high aspect ratios are achievable for nearly any cross-sectional shape (Fig. 3). This so-called advanced silicon etching (ASE) process achieves etching velocities in the order of $0.2 \mathrm{~mm}$ per hour. The ASE dry-etching method has, of course, limitations concerning e.g. material selection and surface smoothness or the brittleness of silicon which makes it nearly impossible to use it directly as a mold insert in micromolding processes. Nevertheless, it is possible to transfer ASE silicon structures into complementary metal structures by electroforming. For a number of applications, ASE is evidently a favorable alter- native to LIGA in manufacturing devices for microreaction technology.

\subsection{Mechanical Micromachining}

In the past few years, impressive progress was achieved in so-called mechanical micromachining utilizing technologies based on ultra-precision machining. Complex three-dimensional microstructures have been generated with shape accuracies in the sub-micrometer range by means of milling, turning and grinding [5][23]. Three- and five-axis ultra-precision micromilling machines are meanwhile available as commercial products. Using diamond tools, an extremely low surface roughness of a few nanometers is achievable for nonferrous materials. Progress has also been made in machining stainless steel by using ultra fine grain hard metal tools and novel technologies like vibration cutting. In addition, mechanical micromachining has been successfully applied with brittle materials. Micromixers, micro heat exchangers and reaction systems have been successfully produced by means of this technology (Fig. 4).

It is evident that there are nearly no limitations concerning the generation of microstructures for chemical microdevices with complex geometries, extremely high aspect ratio and high precision from a wide variety of materials by means of mechanical micromachining. Restrictions may exist in manufacturing closely packed channels or other structures because of the finite size of the tools. Also manufacturing costs may become a problem in mass fabrication but in such a case mechanical micromachining may be helpful for manufacturing mold inserts for mass fabrication by means of micromolding. Moreover, there are further mechanical methods for high volume production like punching and embossing which have in the meantime been successfully applied in fabricating e.g. micro heat exchangers.

An interesting alternative to standard mechanical micromilling, turning, drilling and grinding methods is micro electro discharge machining (EDM) which is virtually unlimited in view of the geometrical shape of the work piece [24]. Material is removed in a discharge between the electrically conductive work piece and an electrode by small sparks in a dielectric fluid like oil or deionized water. An important advantage in micromachining is that the forces acting on the work piece in EDM are extremely low. Disadvantages of micro EDM are a relatively large surface roughness, limitations in miniaturization because of the finite size of the electrodes and the spark gap in the electrical discharge and 
very long machining times so that this method is essentially used to manufacture mold inserts or prototypes.

The methods of mechanical micromachining and micro EDM have been extensively applied for the fabrication of components like micro heat exchangers, mixers and reaction channels as well as chemical microsystems with integrated heat exchange, reaction, mixing and distribution elements (Fig. 5).

\subsection{Micromachining by Means of Laser Radiation}

Micro fabrication by means of laser radiation covers a wide range of different methods [25]. On the one hand, these are processes where material is removed in an intense electromagnetic field by melting, evaporation, decomposition, photo ablation or a combination of these phenomena. On the other hand, generating processes exist where structures are built up from liquid resins, laminated layers, or powders using e.g. photochemically induced cross-linking of organic compounds in stereo lithography or powder solidification by laser sintering. In addition, welding by means of laser radiation is of major importance for connection and assembly of microdevices.

There are no restrictions worth mentioning concerning materials in micromachining by laser radiation which is a real advantage for chemical microdevices (Fig. 6). Limitations rather exist in achieving critical dimensions below $10 \mu \mathrm{m}$ and low surface roughness. Removal of material is also often connected with the generation of debris which reduces accuracy. Since laser-based microfabrication processes, except lithography, are essentially serial rather than parallel machining methods, their productivity is comparatively low. Nevertheless, they offer a huge potential in rapid prototyping.

Laser-based micromachining processes have been applied to date only on a relatively small scale for manufacturing chemical microdevices [26]. This will probably change relatively soon since rapid prototyping will become more and more important for developing novel microreaction devices.

\section{Conclusions}

Nearly all major chemical, chemical engineering, and pharmaceutical companies are now interested or even active in analyzing the potential of microreaction technology. It offers fundamentally novel opportunities to save direct costs in the areas of development, investment, operation and maintenance as well as to reduce indirect

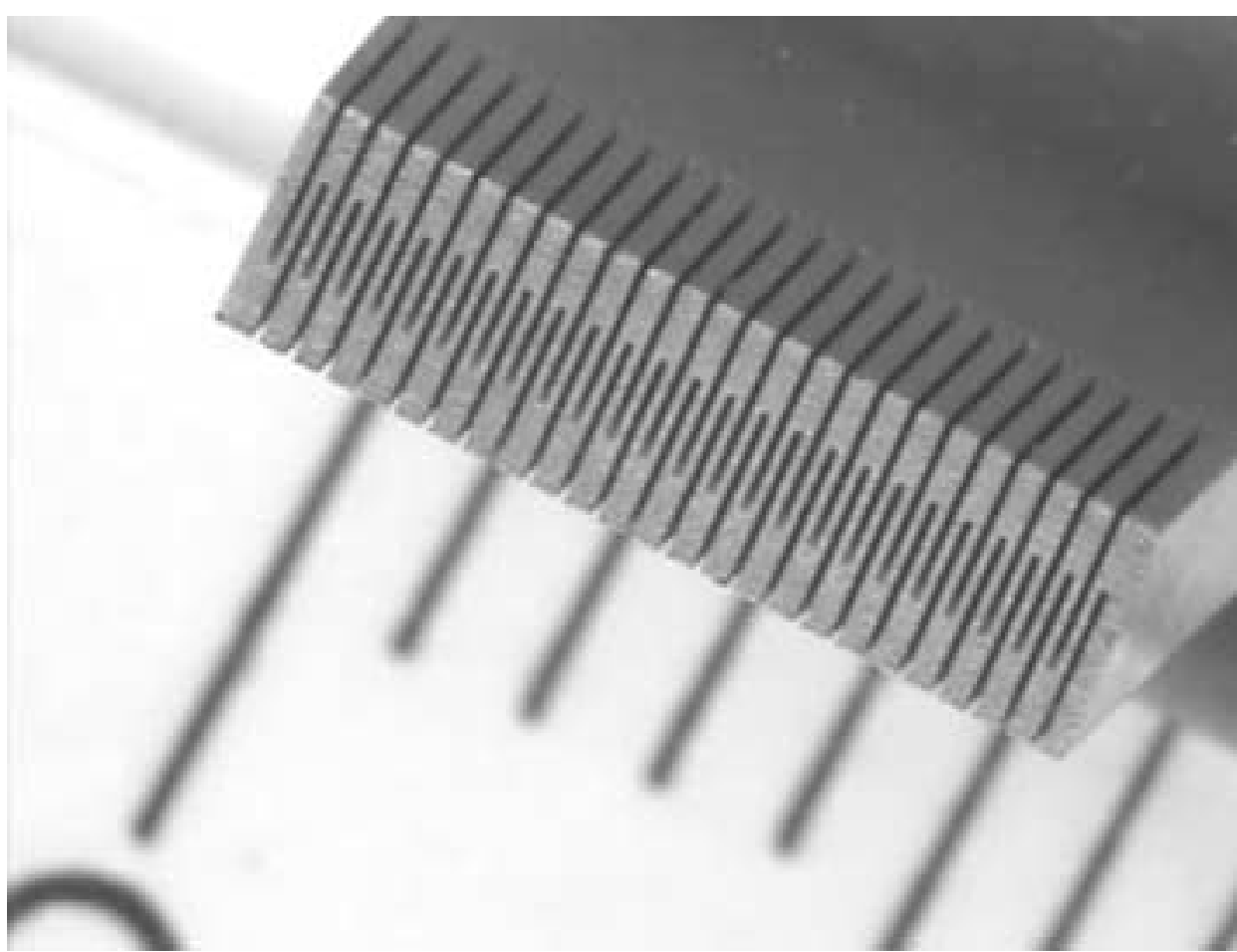

Fig. 5. Micromixing element generated by micro electro discharge machining (Source: Ehrfeld Mikrotechnik, Zumtobel)

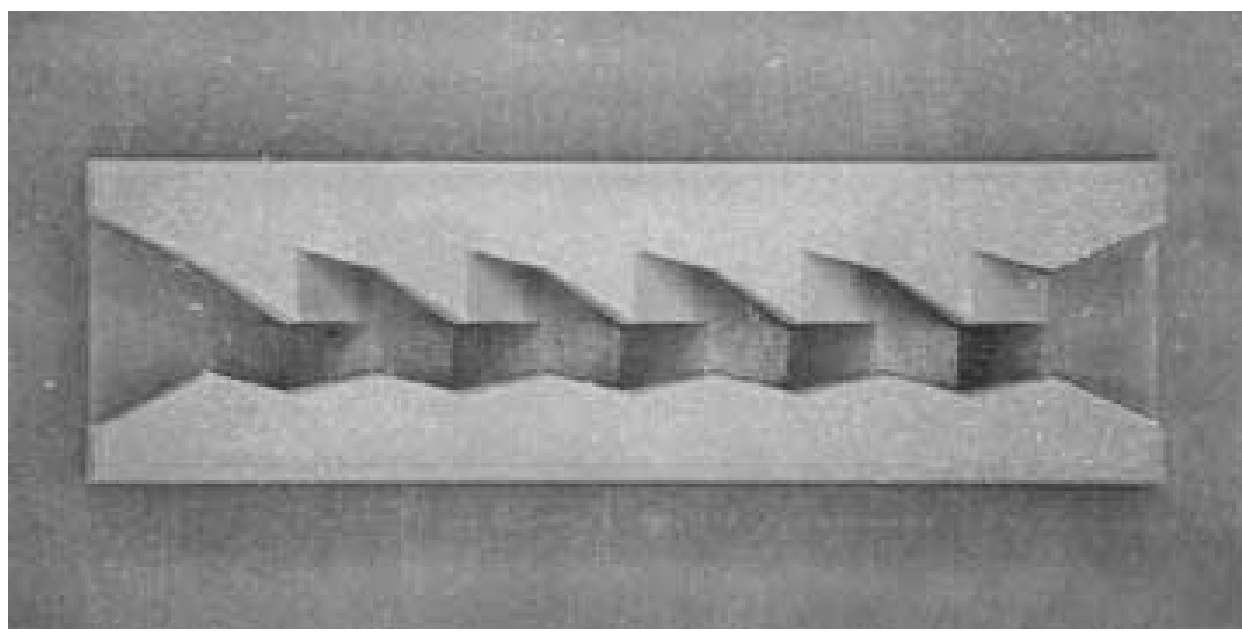

Fig. 6. Part of a static micromixer manufactured by laser ablation from aluminium oxide (Source: Ehrfeld Mikrotechnik, Heidelberg Instruments Microtechnologies)

follow-up expenditures in connection with storage and transport by realizing a sustainable distributed production. In particular, microreaction technology allows research results to be transferred much faster into commercial production and to react more quickly on changes in demand or market trends. Powerful three-dimensional micro fabrication technologies will meet nearly all requirements concerning geometries as well as materials of microreaction devices in prototyping and mass fabrication.

Essential progress is to be expected from the introduction of so-called modular microreaction systems comprising single functional elements for reactions, unit operations, transport, measurement and control. The modules can be arranged and connected in a wide variety of configurations and serve as a toolbox for realizing development platforms similar to microplants (Fig. 7). By means of such platforms the optimum operation conditions of chemical processes as well as favorable plant configurations can be determined and novel reaction routes can be tested. Since the microplants are usually set up for continuous operation, they have a comparatively high productivity and can be directly utilized for small-scale production of special chemi- 
cals.

The implementation of a novel technology is always a tedious task. It is necessary to prove carefully the potential advantages, to develop a sufficiently broad scientific basis, to implement reliable and cost-effective fabrication of chemical microdevices on an industrial scale, to gain experience in design, construction and operation of microreaction plants, and to demonstrate finally real commercial success. However, it is only a question of time. The progress made in other areas of microtechnology like IT and automotive applications will further extend the technological basis of microreaction systems and have a positive impact on decision makers since they become more and more familiar with the fact that miniaturization and integration is a strategy of success.

Received: September 11, 2002

[1] W. Ehrfeld, U. Ehrfeld, S. Kiesewalter, 'Progress and Profit through Microtechnologies', Proc. VDE World Microtechnologies Congress, MICRO.tec 2000, vol. 1, 2000, p. 9-17.

[2] Market analysis for micro systems II, 2000-2005, A NEXUS Task Force Report, 2002.

[3] Bundesministerium für Bildung und Forschung: Förderkonzept Mikrosystemtechnik 2000+, Bonn, 2000.

[4] W. Ehrfeld, V. Hessel, V. Haverkamp, 'Microreactors', Ullmann's Encyclopedia of Industrial Chemistry, 6th ed, Wiley-VCH, Weinheim, 1999.

[5] K. Schubert, W. Bier, G. Linder, D. Seidel, 'Herstellung und Test von kompakten Mikrowärmeübertragern', Chem.-Ing.Tech. 1989, 61. 172-173.

[6] G. Wiessmeier, K. Schubert, D. Hönicke, 'Monolithic Microreactors Possessing Regular Mesopore Systems for the Successful Performance of Heterogeneously Catalysed Reactions', in Proc. 1st Int. Conf. Microreaction Technology, Ed. W. Ehrfeld, Springer-Verlag, Berlin, Heidelberg, New York, 1998, p. 20-26.

[7] K.F. Jensen, I-Ming Hsing, R. Srinivasan, M.A. Schmidt, M.P. Harold, J.J. Lerou, J.F. Ryley, 'Reaction Engineering for Microreactor Systems', in Proc. 1st Int. Conf. Microreaction Technology, Ed. W. Ehrfeld, Springer-Verlag, Berlin, Heidelberg, New York, 1998, p. 2-9.

[8] K.-P. Jäckel, 'Microreaction Technology Vision and Reality', Plenary Lecture, ACHEMA 2000, Frankfurt, 2000.

[9] A.I. Stankiewicz, J.A. Moulijn, 'Process Intensification: Transforming Chemical Engineering', Chemical Engineering Progress 2000, 96, 22-33.

[10] A. Green, B. Johnson, A. John, 'Process Intensification Magnifies Profits', Chemical Engineering Dec. 1999, p. 66-73.

[11] M. Wood, A. Green, 'A Methodological Ap-

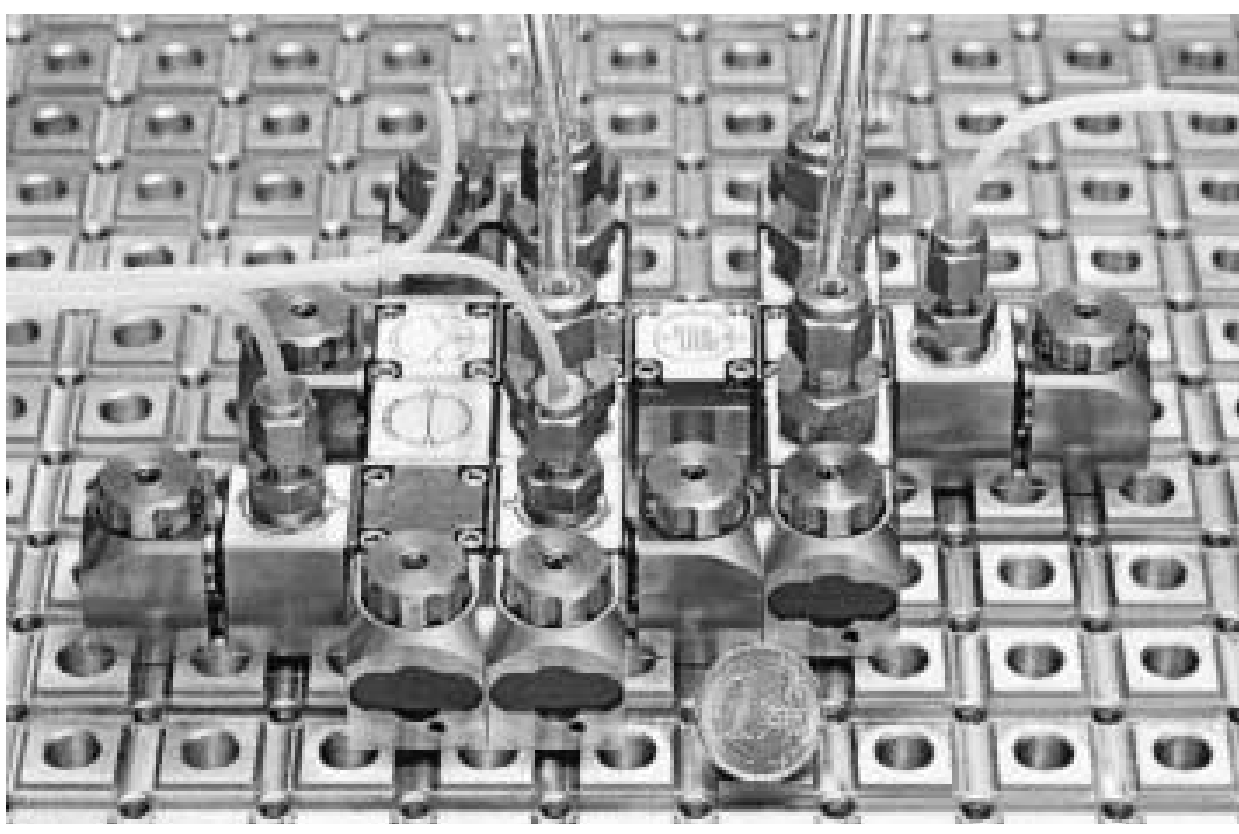

Fig. 7. Modular microreaction system consisting of functional elements for reactions and unit operations arranged on a base plate. The cube-shaped modules of stainless steel with built-in microstructures have a side length of $25 \mathrm{~mm}$ and can be operated at pressures up to 100 bar. (Source: Ehrfeld Mikrotechnik)

proach to Process Intensification', IchemE Symposium Series No. 144, 1998, p. 405-416.

[12] W. Ehrfeld, U. Ehrfeld, 'Microfabrication for Process Intensification', in Proc. 5th Int. Conf. Microreaction Technology, Eds. M. Matlosz, W. Ehrfeld, J.P. Baselt, Springer-Verlag, Berlin, Heidelberg, New York, 2001, p. 3-129.

[13] B. Jandeleit, D.J. Schaefer, T.S. Powers, H.W. Turner, W.H. Weinberg, 'Combinatorial Materials Science and Catalysis', Angew. Chemie, Int. Ed. 1999, 38, 2494-2532.

[14] P. Claus, D. Hönicke, T. Zech, 'Miniaturization of Screening Devices for the Combinatorial Development of Heterogeneous Catalysts', Catalysis Today 2001, 67, 319-339.

[15] J. Mayer, M. Fichtner, D. Wolf, K. Schubert, 'A Microstructured Reactor for the Catalytic Partial Oxidation of Methane to Syngas', in Proc. 3rd Int. Conf. Microreaction Technology, Ed. W. Ehrfeld, Springer-Verlag, Berlin, Heidelberg, New York, 2000, p. 187-196.

[16] H. Löwe, W. Ehrfeld, V. Hessel, T. Richter, J. Schiewe, 'Micromixing Technology', Proc. 4th Int. Conf. Microreaction Technology, AIChE Spring National Meeting, Atlanta, GA, 2000.

[17] U. Hagendorf, M. Jänicke, F. Schüth, K. Schubert, M. Fichtner, 'A Pt/ $\mathrm{Al}_{2} \mathrm{O}_{3}$ Coated Microstructured Reactor/Heat Exchanger for the Controlled $\mathrm{H}_{2} / \mathrm{O}_{2}$ Reaction in the Explosion Regime', Proc. 2nd Int. Conf. Microreaction Technology, p. 81-87, AIChE Spring Meeting, New Orleans, LA, 1998.

[18] W. Ehrfeld, D. Münchmeyer, 'Three-dimensional Microfabrication Using Synchrotron Radiation', Nucl. Inst. Meth.
Phys. Res. 1991, A303, 523-531.

[19] M. Koehler, 'Ätztechniken', in 'Handbuch Mikrotechnik', Ed. W. Ehrfeld, Carl Hanser Verlag, München, 2001, p. 279-322.

[20] A. Freitag, T.R. Dietrich, R. Scholz, 'Glass as a Material for Microreaction Technology', Proc. 4th Int. Conf. Microreaction Technology, p. 48-54, AIChE Spring National Meeting, Atlanta, GA, 2000.

[21] I. W. Rangelow, R. Kassing, 'Silicon Microreactors made by Reactive Ion Etching', Proc. 1st Int. Conf. Microreaction Technology, Springer-Verlag, Berlin, Heidelberg, New York, 1998, p. 169-174.

[22] F. Laermer, A. Schilp (Robert Bosch $\mathrm{GmbH})$, 'Method of Anisotropically Etching Silicon', US Patent No. 5501893, 1996.

[23] M. Weck, 'Ultraprecision Machining of Microcomponents', in Proc. Int. Seminar on Precision Engineering and Microtechnology, Ed. M. Weck, Aachen, 2000.

[24] F. Michel, W. Ehrfeld, O. Koch, H.-P. Gruber, 'EDM for Microfabrication - Technology and Applications', in: Proc. Int. Seminar on Precision Engineering and Microtechnology Ed. M. Weck, Aachen, 2000.

[25] A. Gillner, T. Klotzbücher, 'Lasermikrobearbeitung', in 'Handbuch Mikrotechnik', Ed. W. Ehrfeld, Carl Hanser Verlag, München, 2001, p. 105-143.

[26] E. Bremus, A. Gillner, D. Hellrung, H. Höcker, F. Legewie, R. Poprawe, M. Wehner, M. Wild, 'Laser Processing for Manufacturing Microfluidic Devices', Proc. 3rd Int. Conf. Microreaction Technology, Springer-Verlag, Berlin, Heidelberg, New York, 2000, p. 187-196. 